\title{
Exosomal MiR-1290 Promotes Angiogenesis of Hepatocellular Carcinoma via Targeting SMEK1
}

\author{
Qiong Wang, ${ }^{1}$ Guanwen Wang, ${ }^{1}$ Lianjie Niu, ${ }^{2}$ Shaorong Zhao, ${ }^{1}$ Jianjun Li, ${ }^{1}$ Zhen Zhang, \\ Huimin Jiang, ${ }^{1}$ Quansheng Zhang, ${ }^{3}$ Hang Wang, ${ }^{1}$ Peiqing Sun, ${ }^{4}$ Rong Xiang, \\ Antao Chang $\mathbb{D}^{1}{ }^{1}$ and Shuang Yang $\mathbb{D}^{1}$ \\ ${ }^{1}$ Tianjin Key Laboratory of Tumor Microenvironment and Neurovascular Regulation, Medical College of Nankai University, \\ Tianjin 300071, China \\ ${ }^{2}$ Tianjin Medical University, Tianjin 300070, China \\ ${ }^{3}$ Tianjin Key Laboratory of Organ Transplantation, Tianjin First Central Hospital, Tianjin 300192, China \\ ${ }^{4}$ Department of Cancer Biology, Wake Forest University School of Medicine, Winston-Salem, NC 27157, USA
}

Correspondence should be addressed to Antao Chang; changantao@nankai.edu.cn and Shuang Yang; yangshuang@nankai.edu.cn Received 18 November 2020; Revised 5 January 2021; Accepted 15 January 2021; Published 29 January 2021

Academic Editor: Liren Qian

Copyright (C) 2021 Qiong Wang et al. This is an open access article distributed under the Creative Commons Attribution License, which permits unrestricted use, distribution, and reproduction in any medium, provided the original work is properly cited.

\begin{abstract}
Hepatocellular carcinoma (HCC), the most common primary liver cancer, relies on the formation of new blood vessel for growth and frequent intrahepatic and extrahepatic metastasis. Therefore, it is important to explore the underlying molecular mechanisms of tumor angiogenesis of HCC. Recently, microRNAs have been shown to modulate angiogenic processes by modulating the expression of critical angiogenic factors. However, the potential roles of tumor-derived exosomal microRNAs in regulating tumor angiogenesis remain to be elucidated. In this study, our miRNome sequencing demonstrated that miR-1290 was overexpressed in HCC patient serum-derived exosomes, and we found that delivery of miR-1290 into human endothelial cells enhanced their angiogenic ability. Our results further revealed that SMEK1 is a direct target of miR-1290 in endothelial cells. MiR-1290 exerted its proangiogenic function, at least in part, by alleviating the inhibition of VEGFR2 phosphorylation done by SMEK1. Collectively, our findings provide evidence that miR-1290 is overexpressed in HCC and promotes tumor angiogenesis via exosomal secretion, implicating its potential role as a therapeutic target for HCC.
\end{abstract}

\section{Introduction}

Hepatocellular carcinoma is the fifth most common cancer and third-leading cause of cancer-related deaths worldwide [1]. HCC is a highly vascularized tumor with frequent intrahepatic and extrahepatic metastasis, which is responsible for the rapid recurrence and poor survival [2]. Therefore, it is urgent to explore the molecular mechanisms of tumor angiogenesis and metastasis, which would provide potential effective therapeutic approaches to improve the survival of HCC patients.

MicroRNAs (miRNAs) are a family of noncoding RNAs, about $22 \mathrm{nt}$ in length, which repress gene expression by pairing to the $3^{\prime}$-untranslated regions of target messenger RNAs (mRNAs) [3]. MiRNAs are involved and play critical roles in a variety of biological processes such as tumor progression [4]. Deregulation of miRNAs contributes to angiogenesis and metastasis of various human cancers including HCC [5-7]. For example, microRNA-26a has been reported to suppress angiogenesis in HCC by targeting HGF-cMet pathway [8]. Recently, emerging evidence has revealed that miRNAs can be secreted into tumor microenvironment via exosomes to mediate the crosstalk between cancer cells and tumor microenvironment [9-12].

Exosomes are small $(40-100 \mathrm{~nm})$ membrane vesicles released into the extracellular environment [13]. It is reported that exosomes contain miRNAs selectively enriched from parent cells [14]. Circulating exosomal miRNAs may have additional advantages as biomarkers over and above 'free' miRNAs. A number of plasma exosomal miRNAs are 
reported as diagnostic, prognostic, or even therapeutic biomarkers in cancer patients [15-17]. For example, exosomal miR-1290 and miR-375 are prognostic markers in castration-resistant prostate cancer [18]. Exosomal miR-210 could be delivered into endothelial cells and directly inhibits the expression of SMAD4 and STAT6, resulting in enhanced HCC angiogenesis [19]. However, the underlying mechanism of exosomal miRNAs in HCC development, especially in tumor angiogenesis of HCC, remains largely unknown.

In this study, by RNA sequencing and qRT-PCR verification, we revealed that miR-1290 was highly expressed in HCC patient serum-derived exosomes. Using gain- and lossof-function analyses, we further demonstrated the proangiogenic function of miR-1290 in mediating the crosstalk between HCCs and tumor endothelial cells. Moreover, we found that SMEK1 was a bona fide target of miR-1290 in endothelial cells. MiR-1290 exerted its proangiogenic function, at least in part, by alleviating the inhibition of VEGFR2 phosphorylation done by SMEK1. Collectively, our findings suggest that exosomal miRNA-1290 might play an important role in the intercellular communication in tumor angiogenesis of HCC.

\section{Materials and Methods}

2.1. Clinical Specimens. Primary HCC tumor samples, paired tumor adjacent tissues, and serum samples of 49 HCC patients and serum samples of 28 healthy individuals were obtained from Tianjin First Central Hospital. Among them, serum samples from six HCC patients and two healthy individuals were subjected to miRNome sequencing. Serum samples and surgically removed tissues were frozen and transported in liquid nitrogen. All of the samples were obtained with written informed consent from patients and approval from the Ethics Committee of Tianjin First Central Hospital and Medical School of Nankai University.

2.2. Experimental Animals. Adult male BALB/c and NODSCID mice were ordered form HuaFuKang Bioscience Co. (Beijing, China), housed under specific pathogen free environmental conditions. All mouse experiments were conducted in accordance with the standard operating procedures approved by the Institute Research Ethics Committee at the Nankai University.

2.3. Exosome Purification. Exosome extraction from serum and cell supernatant samples using the exosome isolation reagent (Ribobio Co., China) were performed according to the manufacturer's protocol. For electron microscopy, the serum was precleared by centrifugation at $300 \times g$ for $5 \mathrm{~min}$, $2000 \times g$ for $10 \mathrm{~min}$, and then at $10000 \times g$ for $60 \mathrm{~min}$. Exosomes were isolated by ultracentrifugation at $100000 \times g$ for $130 \mathrm{~min}$, followed by PBS washing under the same ultracentrifugation conditions. Exosomes were resuspended in $100 \mu \mathrm{l}$ PBS, fixed with $2 \%$ paraformaldehyde, loaded on 200mesh Formvar-coated grids, contrasted, and embedded for imaging.
2.4. Cell Lines and Reagents. Human umbilical vein endothelial cells (HUVECs) and human HCC cell lines Hep3 B and HepG2 were obtained from ATCC. HCC cell line SMMC-7721, PLC/PRF/5, and normal human hepatocyte cell line L-02 were purchased from the Chinese Academy of Sciences (Shanghai, China). Hep3 B, HepG2, and PLC/PRF/ 5 cells were cultured in MEM containing 10\% fetal bovine serum (FBS), $100 \mathrm{U} / \mathrm{ml}$ penicillin/streptomycin, and $100 \mu \mathrm{g} /$ $\mathrm{ml}$ nonessential amino acids. SMMC-7721 cells were grown in DMEM containing 10\% FBS and $100 \mathrm{U} / \mathrm{ml}$ penicillin/ streptomycin. L-02 and HUVECs were cultured in RPMI1640 containing $10 \% \mathrm{FBS}$ and $100 \mathrm{U} / \mathrm{ml}$ penicillin/streptomycin. MiR-1290 mimics, inhibitors, agomir, antagomir, and miR-1290 mimic-FAM were designed and synthesized by Ribobio Co. The sequences of miR-1290 mimic and agomir are UGGAUUUUUGGAUCAGGGA and the sequences of miR-1290 inhibitor and antagomir are UCCCUGAUCCAAAAAUCCA. The miR-1290 agomir and antagomir were all nucleotides with a $2^{\prime}$-O-methyl modification. The miR-1290 mimic and inhibitor were transiently transfected with lipofectamine 2000 (Invitrogen) according to the manufacture's protocol.

2.5. Cell Viability Assays. For cell viability assays, HUVECs were seeded in 96-well plates in $100 \mu \mathrm{l}$ of medium, followed by transfection with $50 \mathrm{nM}$ miR-1290 mimics. Five parallel wells were assigned for each group. At $0,24,36,48$, and $60 \mathrm{~h}$ after transfection, CCK- 8 solution $(10 \mu \mathrm{l})$ was added to each well, and absorbance values were measured at $450 \mathrm{~nm}$ after incubation for $1.5 \mathrm{~h}$ at $37^{\circ} \mathrm{C}$.

2.6. RNA Extraction and Quantitative Real-Time PCR ( $q R T$ $P C R$ ). Tissues were grinded using a homogenizer in Trizol reagent (Invitrogen, USA). Cells were collected, washed, and lysed in Trizol reagent, and total RNA was isolated following the manufacturer's instructions. qRT-PCR was performed using a standard SYBR-Green PCR kit protocol. Primers are listed in Table S1.

2.7. Western Blotting. Western blotting was performed in accordance with a previous protocol [20]. Briefly, proteins were loaded on $5-12 \%$ tris-acrylamide gels and membranes were blotted with specific antibodies. The primary antibodies used in this study were SMEK1 (ab70635, Abcam), pVEGFR2 (\#4991, Cell Signaling Technology), and VEGFR2 (\#9698, Cell Signaling Technology) at a dilution of 1:1000. Bands were detected using a Gel imaging system (SYNGENE).

2.8. Plasmid Construction and Transfection. Human SMEK1 cDNA was cloned from HUVECs and ligated into pLVEF1 $\alpha$-MCS-IRES-Bsd vector (Biosettia Inc.). SMMC-7721, Hep3 B, and HUVECs $\left(3 \times 10^{5}\right.$ cells/well $)$ were seeded in 6well plates, incubated overnight, and then transfected with miR-1290 mimics using lipofectamine 2000 (Invitrogen) and Opti-MEM (Corning) according to the manufacturer's instruction. Primers are listed in Table S2. 
2.9. Cell Migration Assays. Cell migration was evaluated by performing wound-healing and transwell assays. For wound-healing assay, cells were seeded in 6-well plates at $2 \times 10^{5}$ cells per well, and after $48 \mathrm{~h}$ transfection, the cell monolayer was scraped using a $10 \mu \mathrm{l}$ tip. The initial gap length and the residual gap length after wounding were calculated based on photomicrographs using ImageJ software. For transwell assay, after transfection for $24 \mathrm{~h}$, cells were plated in 24 -well plates at $5 \times 10^{4}$ in the upper 8 $\mu \mathrm{m}$ chambers (BD bioscience). Medium containing $10 \%$ FBS in the lower well and 2\% FBS in the upper chamber served as the chemoattractant. After another $16 \mathrm{~h}$ incubation, cells on the upper surface of the membrane were scraped off and those on the bottom were stained with crystal violet. These cells were photographed using an optical microscope.

2.10. Tube Formation and Matrigel Plug Assays. For tube formation assay, a prechilled 48-well plate was coated with $150 \mu \mathrm{l}$ of Matrigel (BD bioscience) and incubated at $37^{\circ} \mathrm{C}$ for $30 \mathrm{~min}$. HUVECs $\left(3 \times 10^{4}\right.$ cells/well $)$ were transfected with miR-1290 and then seeded on this plate. After $5 \mathrm{~h}$, photographs were taken, and the tubes were counted.

For in vivo Matrigel plug assay, male $\mathrm{BALB} / c$ mice were administered a subcutaneous injection of $750 \mu \mathrm{l}$ of mixture containing $500 \mu \mathrm{l}$ Matrigel and $250 \mu \mathrm{l}$ EBM2 medium with or without $10 \mathrm{nmol}$ miR-1290 agomir (3 mice for each group). After 10 days, the Matrigel plugs were imaged and snap frozen in the presence of optimum cutting temperature (OCT) medium before sectioning. Frozen Matrigel sections $(8 \mu \mathrm{m})$ were fixed in cold methanol and immunostained with a CD31 antibody (ab28364, Abcam, USA).

2.11. Immunofluorescence Staining. Slides were blocked with $2 \%$ BSA and then incubated with an anti-CD31 antibody (ab28364, Abcam) for $1 \mathrm{~h}$. After fluorophore-conjugated secondary antibody incubating and PBS washing, slides were incubated with DAPI (Invitrogen) for 2 min. Images were obtained using a microscope (Olympus IX73).

2.12. Dual Luciferase Reporter Assay. HUVECs were cultured in 24-well plates at a density of $2 \times 10^{5}$ cells/well overnight, which was followed by cotransfection with miR1290 mimics, pmirGLO, and the pRL-TK plasmid. After $36 \mathrm{~h}$ of transfection, the inhibition of miR-1290 was quantified as the ratio of firefly luciferase activity to Renilla luciferase activity in each well.

2.13. Tumor Xenografts. Male NOD-SCID mice (6 weeks old) were separated randomly into two groups ( $n=6$ each), and $2 \times 10^{6}$ SMMC-7721 cells were inoculated subcutaneously into each mouse, and tumors were allowed to grow for 10 days. Tumors were peritumorally treated with $10 \mathrm{nmol}$ miR-1290 antagomir or miRNA antagomir negative control
(NC) every three days, and tumor volume was measured with calipers once every 3 days.

2.14. Immunohistochemistry (IHC). IHC staining was performed using paraffin-embedded human HCC tissues and mouse xenografts tumors. These tissues were probed separately with an antibody against SMEK1 (ab70635, Abcam), pVEGFR2 (\#4991, Cell Signaling Technology), Ki67 (ab16667, Abcam), cleaved-caspase 3 (\#9664, Cell Signaling Technology), and CD31 (ab28364, Abcam) at a 1:100 dilution.

2.15. Statistical Analysis. Statistical analyses were performed using SPSS 23.0 software; the data from all experiments are presented as means \pm SD and represent three independent experiments. A paired $t$-test was used to compare gene expression in tumor and adjacent nontumor tissue samples. Where appropriate, a Student's $t$-test for unpaired observations was applied. A value of $p<0.05$ was considered significant.

\section{Results}

3.1. MiR-1290 Is Highly Expressed in HCC Patient SerumDerived Exosomes, Tumor Tissues, and HCC Cell Lines. To uncover miRNAs that are dysregulated in HCC patient serum-derived exosomes, we collected serum exosomes from the healthy individuals and HCC patients to perform RNA-seq (Figure 1(a)). We totally identified 599 differently expressed miRNAs (Table S3). After filtering ( $\mid \log 2$ fold change $\mid \geq 1, p$ value $<0.05$ ), we acquired 5 upregulated and 72 downregulated miRNAs (Figure 1(b)). Since upregulated miRNAs are convenient biomarkers and potential therapeutic targets for HCC, we moved on to validate the upregulated miRNAs, including miR-1290, miR-1246, miR4497, miR-1261, and miR-7641. Among these upregulated miRNAs, we pay our attention to miR-1290 because miR1290 is the topmost upregulated miRNA (Figure 1(c)). To confirm the upregulation of miR-1290 in HCC patients, we further measured its expression in serum-derived exosomes from 28 healthy individuals and 49 HCC patients. The results revealed that the expression of miR-1290 was significantly increased in serum exosomes derived from 49 HCC patients as compared to those from 28 healthy individuals (Figure 1(d)). We also detected the expression level of miR1290 in these 49 frozen HCC tissues and paired cancer adjacent tissues. Our qRT-PCR results demonstrated that miR-1290 expression was remarkably elevated in HCC tissues (Figure 1(e)). In addition, we assessed the expression of miR-1290 in 4 HCC cell lines, including HepG2, Hep3 B, SMMC-7721, and PLC/PRF/5 and their exosomes derived from the medium. An immortalized liver cell line L-02 was used as a control. Our results showed that the expression levels of miR-1290 were relatively higher in HepG2 and SMMC-7721 cells as well as their exosomes (Figure 1(f)). Taken together, the above observations indicated that miR1290 is overexpressed in HCCs and cancer-cell-secreted exosomes. 


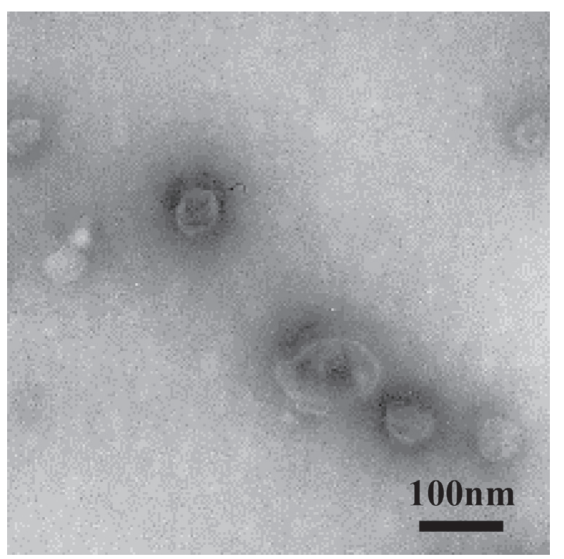

(a)

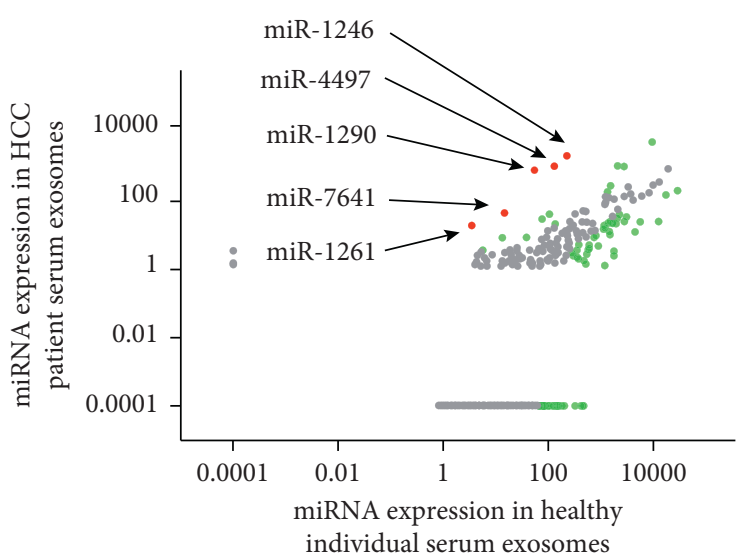

(b)

\begin{tabular}{|ccccc}
\hline \multicolumn{1}{c}{ miRNA ID } & $\begin{array}{c}\text { Expression in healthy } \\
\text { individual serum exosomes }\end{array}$ & $\begin{array}{c}\text { Expression in patient } \\
\text { serum exosomes }\end{array}$ & $\begin{array}{c}\text { Log2 (fold } \\
\text { change) }\end{array}$ & $p$ value \\
\hline has-miR-1290 & 54.622 & 539.6257 & 3.3044 & $1.02 E-18$ \\
\hline has-miR-1246 & 225.8127 & 1377.517 & 2.6089 & $2.92 E-17$ \\
has-miR-4497 & 131.1397 & 698.088 & 2.4123 & $1.53 E-16$ \\
has-miR-1261 & 3.5064 & 14.1103 & 2.0087 & $8.76 E-07$ \\
has-miR-7641 & 14.7269 & 32.0266 & 1.1208 & $7.44 E-10$ \\
\hline
\end{tabular}

(c)

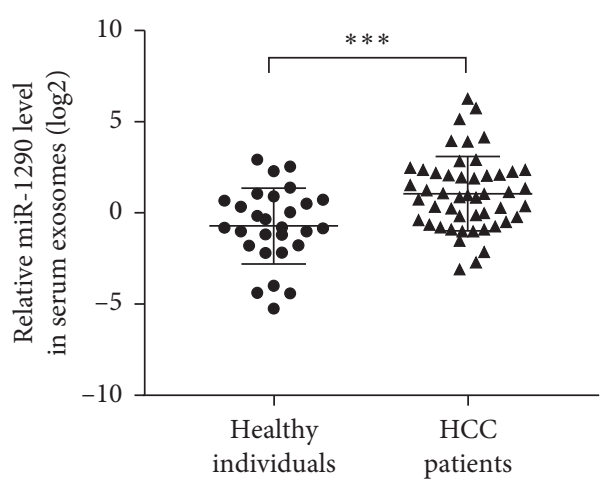

(d)

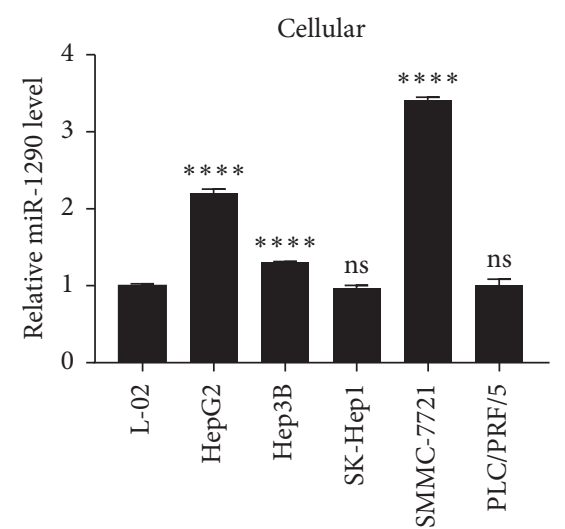

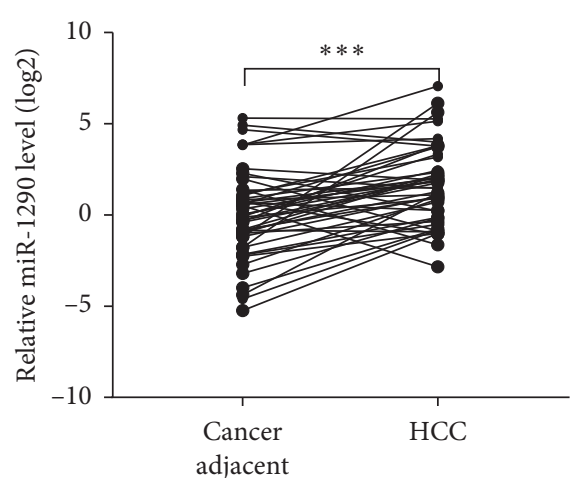

(e)

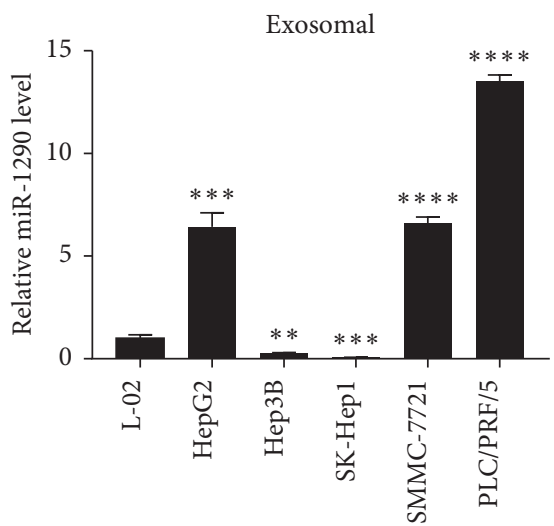

$(\mathrm{f})$

FIGURE 1: MiR-1290 is upregulated in serum-derived exosomes from HCC patients and cell lines. (a) Images of exosomes derived from HCC patient serum were captured by electron microscope. (b) The scatter diagram shows the differentially expressed miRNAs in exosomes derived from HCC patients and healthy individuals by RNA-sequencing. Red dots represent the upregulated miRNAs, green dots represent the downregulated miRNAs, and gray dots represent the unchanged miRNAs. (c) Detailed information of the significantly upregulated miRNAs are listed. (d, e) The expression levels of exosomal miR-1290 in serum from healthy individuals and HCC patients (d) and in HCC tissues and paired tumor adjacent tissues (e) were detected by qRT-PCR. (f) Cellular (left panel) and exosomal (right panel) miR-1290 levels in HCC and normal hepatic cell lines were assessed by qRT-PCR. ${ }^{* *} p<0.01 ;{ }^{* * *} p<0.001 ;{ }^{* * * *} p<0.0001$. Ns, not significant. 
3.2. HCC-Derived Exosomal MiR-1290 Targets Endothelial Cells to Promote Tumor Angiogenesis. Next, we moved to validate whether HCC-derived exosomal miR-1290 could remodel the tumor microenvironment through targeting the endothelial cells and tumor angiogenesis. To this end, we firstly detected the expression of miR-1290 in HepG2, SMMC-7721, and HUVECs. As shown in Figure 2(a), the expression of miR-1290 was significantly increased in HepG2 and SMMC-7721 cells as compared to that in HUVECs (Figure 2(a)). To further test whether the cancer cell-derived exosomes can interact with HUVECs, we collected exosomes from HepG2 and SMMC-7721 cells labeled by DiI and incubated them with HUVECs labeled by $\mathrm{DiO}$. We observed a colocalization of $\mathrm{DiI}$ and $\mathrm{DiO}$ signals in HUVECs cocultured with HCC-derived exosomes, indicating that cancer-cell-secreted exosomes can interact with endothelial cells (Figure 2(b)). We also analyzed whether miR-1290 can be transported from cancer cells to HUVECs as an exosome cargo. To do so, we transfected SMMC-7721 cells with FAM-labeled miR-1290 mimic. Exosomes were then derived from the medium to incubate with HUVECs. The results confirmed that FAMlabeled miR-1290 mimic were detected in HUVECs (Figure 2(c)), demonstrating that HCC-secreted miR-1290 can be transported from cancer cells to HUVECs via exosomes.

Additionally, we examined whether miR-1290 altered the function of HUVECs. Firstly, we performed CCK- 8 assay to detect the cell viability of HUVECs in the presence of miR-1290 mimic and inhibitor, respectively. As shown in Figure 2(d), overexpression of miR-1290 mimic significantly enhanced cell viability at $48-60 \mathrm{~h}$ after transfection; however, miR-1290 inhibitor performed the opposite effect to reduce cell viability. Moreover, we measured the tube formation ability of HUVECs in response to miR-1290 mimic or inhibitor transfection. We found that the capacity of HUVECs to form tube-like structures on the Matrigel was increased by miR-1290 mimic but decreased by miR-1290 inhibitor (Figure 2(e)). Wound-healing and Transwell assays further revealed that miR-1290 mimic significantly accelerated HUVECs migration, whereas miR-1290 inhibitor decreased this effect (Figures 2(f) and 2(g)). These results collectively indicated that miR-1290 might promote endothelial function and tumor angiogenesis in vitro.

3.3. MiR-1290 Promotes Tumor Angiogenesis In Vivo. To determine whether miR-1290 is involved in tumor angiogenesis in vivo, Matrigel plug assays were performed in $\mathrm{BALB} / \mathrm{c}$ mice. As shown in Figure 3(a), the numbers of newly generated blood vessels in Matrigel plugs were increased in mice treated with miR-1290 agomir, compared to the NC agomir group. Immunofluorescent staining of CD31 further revealed that treatment with miR-1290 agomir resulted in significantly increased microvessel density in the plug sections.

On the contrary, we established a SMMC-7721 xenograft tumor model in NOD-SCID nude mice using miR-1290 and
NC antagomirs, respectively. The results indicated that tumor volumes and weights were significantly decreased in mice peritumorally injected with miR-1290 antagomir compared with those of mice injected with NC antagomir (Figures 3(a)-3(d)). Immunohistochemical analysis also revealed the downregulation of Ki67 and the upregulation of cleaved-caspase 3 in miR-1290 antagomir-treated tumors, indicating decreased cell proliferation and increased cell apoptosis (Figure 3(e)). Importantly, reduced angiogenesis was observed in miR-1290 antagomir-treated tumors, as assessed by CD31 staining (Figure 3(f)). Moreover, we divided 49 HCC patients into two groups based on their miR1290 expression levels. Clinical association analysis showed that high level of miR-1290 was correlated with bigger tumor size and advanced clinical stages (Table 1). Notably, IHC staining for CD31 revealed that the microvessel density was increased in tumors with high miR-1290 expression compared to tumors with lower miR-1290 expression (Figure 3(g)), which is consistent with our results showing that miR-1290 acts as an oncogene to promote tumor angiogenesis in vitro and in vivo.

3.4. SMEK1 Is a Direct Target of MiR-1290 in HUVECs. To investigate the potential target genes of miR-1290 in the angiogenic process, we performed a miRNA target gene prediction with Targetscan database and identified 10 candidate genes that have been reported to function in the regulation of angiogenesis (Figure 4(a)). We then performed qRT-PCR to detect the mRNA levels of these 10 candidate genes in HUVECs transfected with miR-1290 mimic. The results demonstrated that all of these miR-1290 potential targets were downregulated by miR-1290 mimic transfection (Figure 4(b)). Moreover, we cloned the $3^{\prime}$ UTR fragments of these genes into a pmirGLO luciferase reporter system, respectively. The results of luciferase assay showed a remarkable repression in the wild-type SMEK1 $3^{\prime}$ UTR reporter in response to miR-1290 mimic treatment in HUVECs (Figure 4(c)). However, this effect was significantly abolished by using the mutant SMEK1 $3^{\prime}$ UTR (Figures 4(d)4(e)). To further confirm the correlation between miR-1290 and SMEK1, we detected the expression of SMEK1 in HUVECs upon transfection with miR-1290 mimic. Our results demonstrated that transfection of miR-1290 mimic downregulated SMEK1 expression at both the mRNA and protein levels in a dose-dependent manner (Figure 4(f)), suggesting that SMEK1 is a direct target of miR-1290. Western blot (Figure S1(a)) and immunohistochemical analysis also revealed the upregulation of SMEK1 (Figure S1(b)) and the downregulation of pVEGFR2 (Figure S1(c)) in the previous miR-1290 antagomir-treated xenografts.

3.5. SMEK1 Reduces the Angiogenic Ability of Endothelial Cells. Considering that SMEK1 has been reported to function as an antiangiogenic factor by suppressing the phosphorylation of VEGFR2 in HUVECs [21], we further examined the endothelial phenotypes in response to 

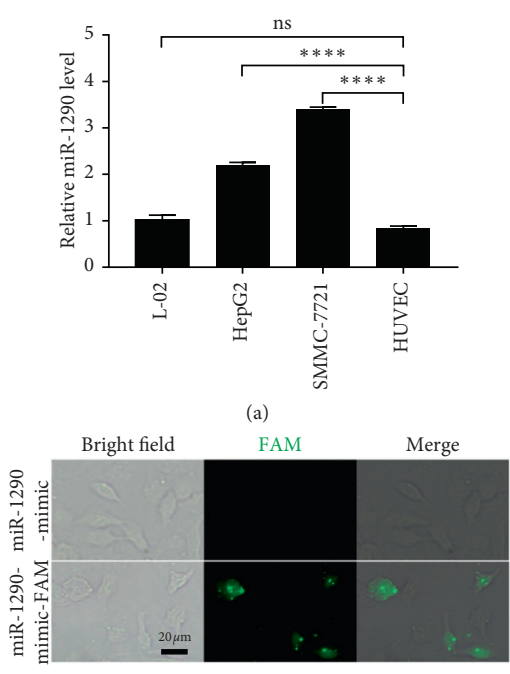

(c)
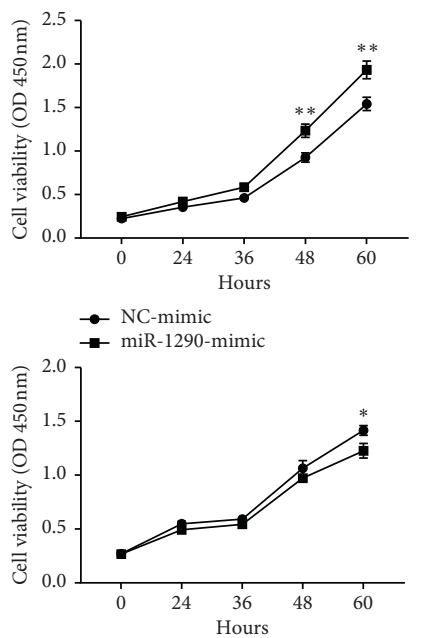

$\rightarrow$ NC-inhibitor

$\rightarrow$ miR-1290-inhibitor
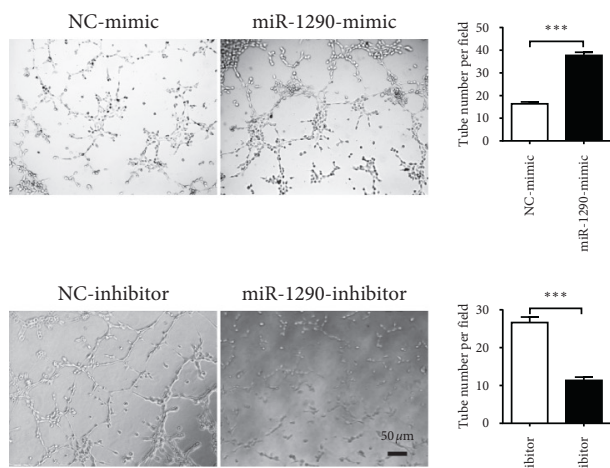

(e)

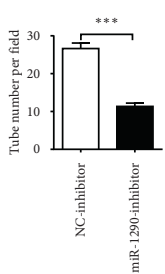

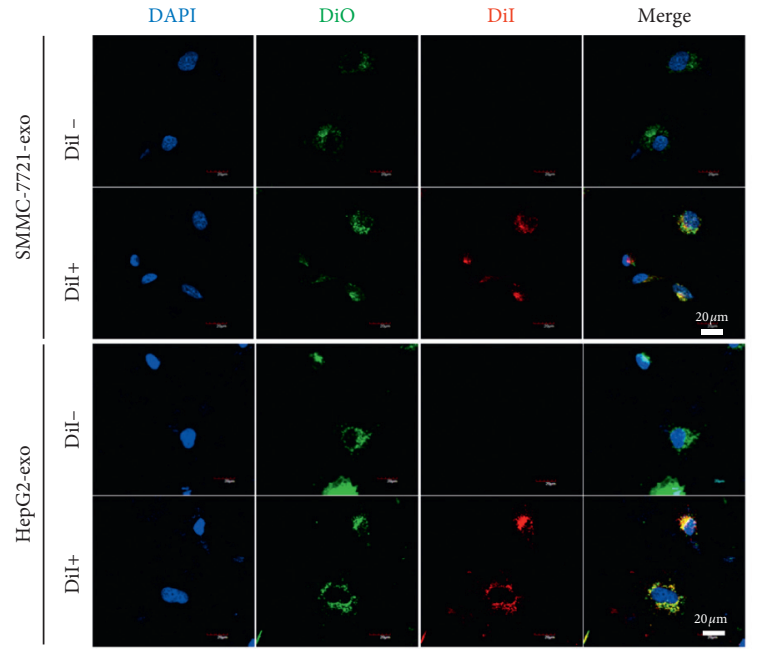

(b)
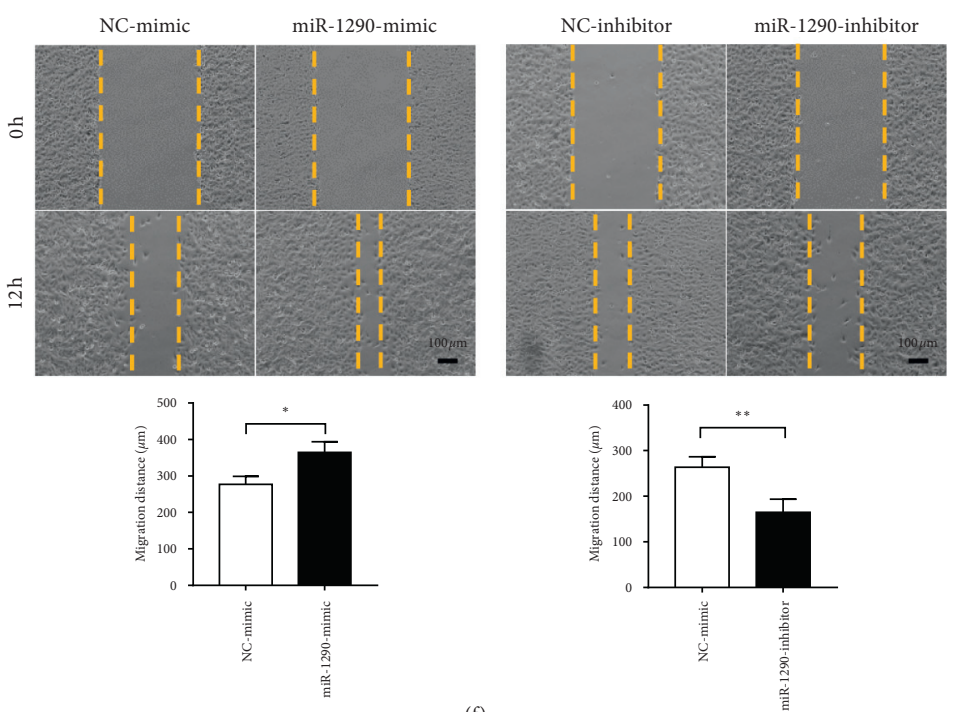

(f)
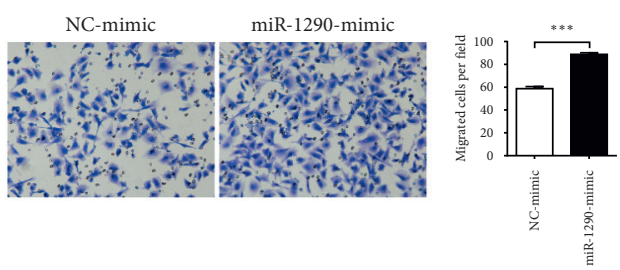

NC-inhibitor

miR-1290-inhibito
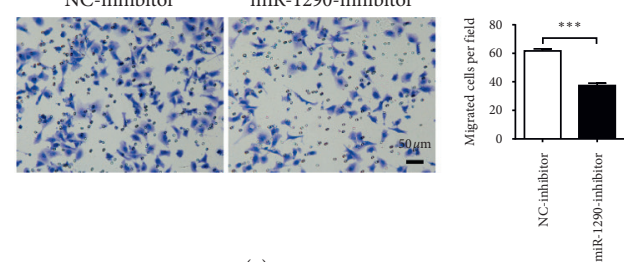

FIgURE 2: Exosomal miR-1290 targets endothelial cells and exerts an angiogenic effect in vitro. (a) The expression level of miR-1290 in HUVECs, HepG2, SMMC-7721, and L-02 cells were detected by qRT-PCR. (b) Exosomes were isolated from the conditioned medium of DiI-labeled (DiI+) or control (DiI-) HCCs and applied to culture with DiO-labeled HUVECs for 24 (h) followed by confocal microscope detection. (c) Exosomes were isolated from the conditioned medium of SMMC-7721 cells that transfected with miR-1290-FAM or miR-1290 mimic and applied to culture HUVECs for $6 \mathrm{~h}$ before detection for fluorescent FAM. (d) Cell viabilities of SMMC-7721 cellstransfected with $50 \mathrm{nM}$ miR-1290 mimic, miR-1290 inhibitor, or the negative control were detected by CCK- 8 assay. (e, f, g) The tube formation (e), woundhealing (f), and Transwell (g) assays of HUVECs were performed in HUVECs that transfected with $50 \mathrm{nM}$ miR-1290 mimic, miR-1290 inhibitor, or the negative control. ${ }^{*} p<0.05 ;{ }^{* *} p<0.01 ;{ }^{* * *} p<0.001 ;{ }^{* * * *} p<0.0001$. Ns = not significant. 

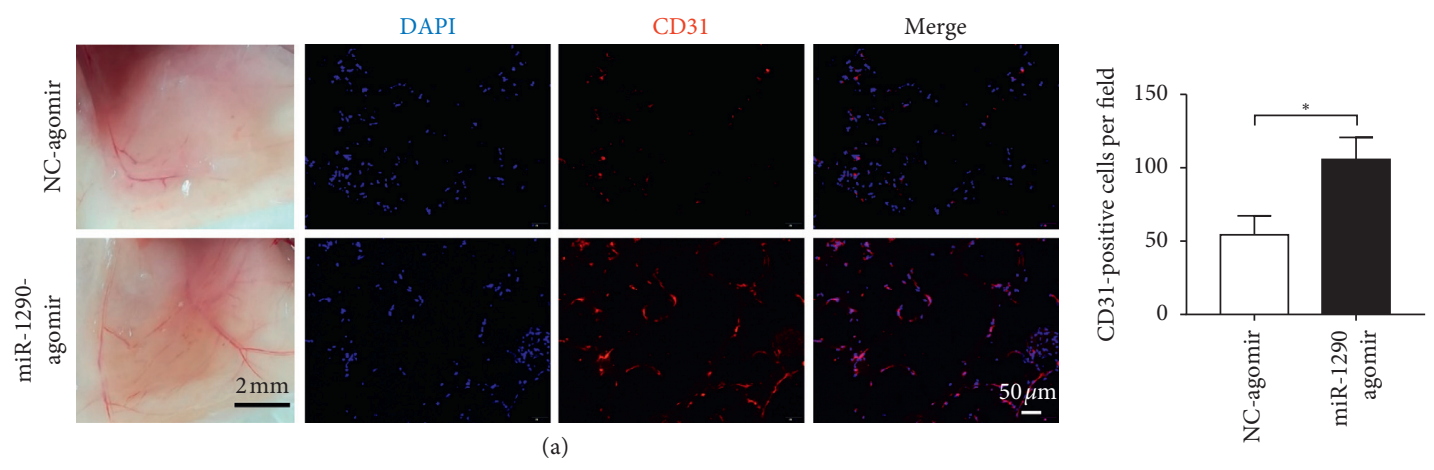

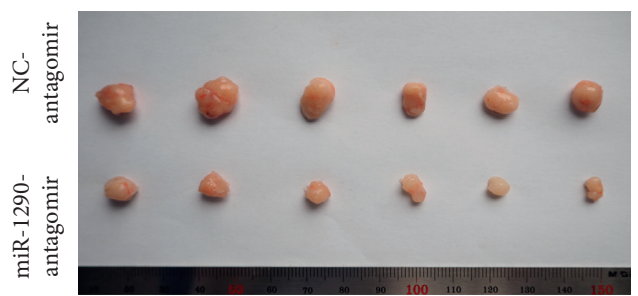

(b)

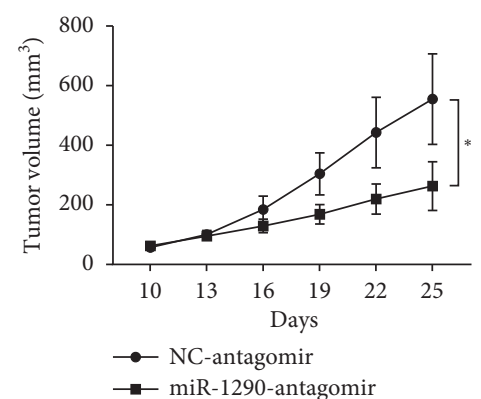

(c)

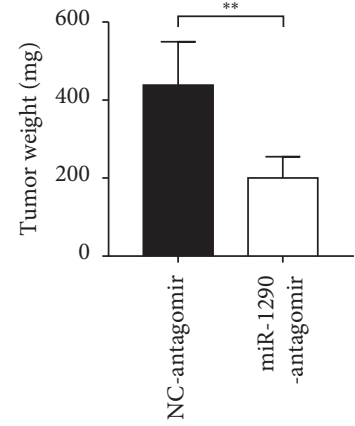

(d)
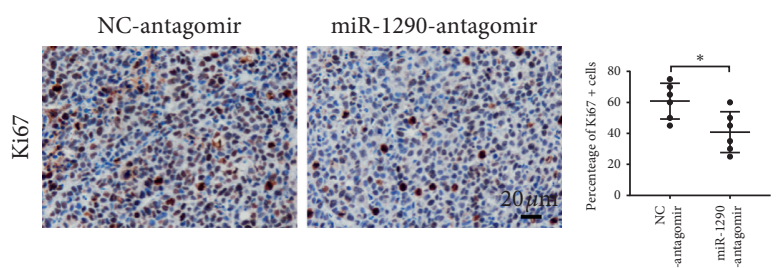

NC-antagomir

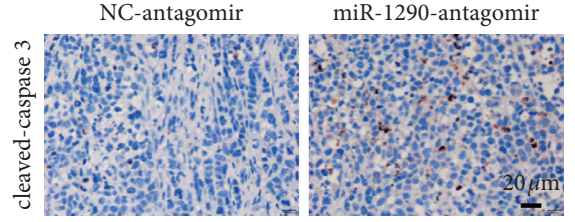

(e)

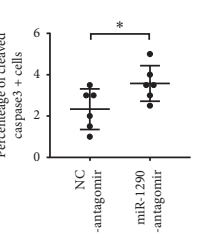

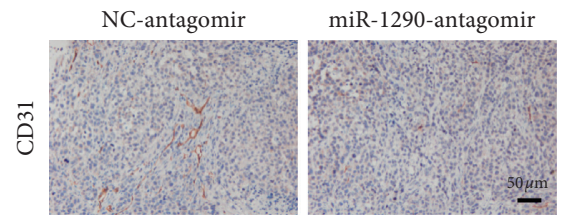

(f)

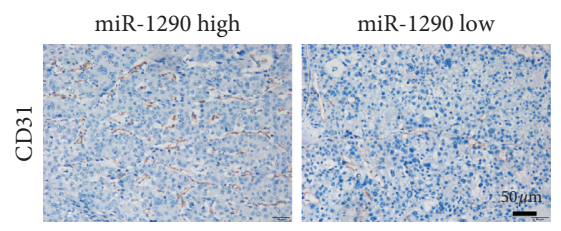

(g)
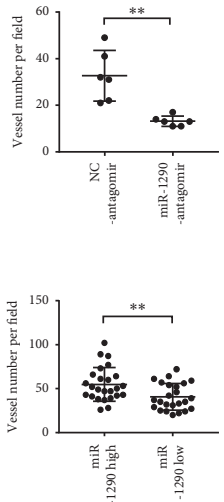

Figure 3: miR-1290 promotes tumor angiogenesis in vivo. (a) Matrigel mixed with miR-1290 agomir or NC agomir were subcutaneously injected into $\mathrm{BALB} / \mathrm{c}$ mice ( $n=3$ each) and then harvested 10 days after inoculation, followed by immunofluorescence staining for CD31. (b) $2 \times 10^{6}$ SMMC-7721 cells were subcutaneously injected into male NOD-SCID mice. Tumor development was allowed to occur for 10

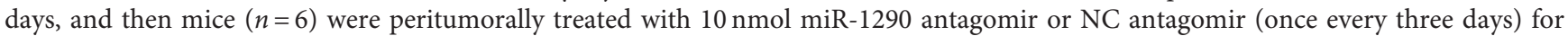
another 15 days. Tumors from miR-1290 antagomir and NC antagomir-treated mice are shown. (c, d) Tumor volume (c) and weight (d) were measured. The proliferation ((e), upper panel), apoptosis ((e), lower panel), and vessel density (f) of the tumors were assessed by IHC stanning for KI67, cleaved-caspase 3, and CD31 separately. (g) 49 HCC patients were divided into two groups based on their miR-1290 expression. The microvessel density of paraffin-embedded tumor sections from these 49 HCC patients was assessed by IHC stanning for CD31. ${ }^{*} p<0.05 ;{ }^{* *} p<0.01$.

SMEK1 interference. qRT-PCR and western blotting assays showed reduced SMEK1 mRNA and protein levels in HUVECs transfected with two independent SMEK1 shRNAs (Figures 5(a) and 5(b)). Notably, we found that the ability of HUVEC to form tube-like structures on the Matrigel was increased by SMEK1 knockdown (Figure 5(c)). Wound-healing and Transwell assays also revealed that depletion of SMEK1 significantly promoted the migration of HUVECs (Figures 5(d) and 5(e)), confirming that SMEK1 might act as a tumor suppressor to inhibit the endothelial function in HCC.

3.6. SMEK1 Mediates MiR-1290-Induced Proangiogenic Phenotypes. Next, to study whether miR-1290 exerts its proangiogenic function through SMEK1, we overexpressed SMEK1 lacking 3'UTR in HUVECs via lentiviral transfection. As shown in Figures 6(a) and 6(b), 
TABLE 1: Correlation between the clinicopathological characteristics and miR-1290 expression in HCC.

\begin{tabular}{|c|c|c|c|c|c|}
\hline \multirow{2}{*}{\multicolumn{2}{|c|}{ Clinicopathologic features }} & \multicolumn{4}{|c|}{ Exosomal miR-1290 } \\
\hline & & $n$ & High & Low & $p$ value \\
\hline \multirow{2}{*}{ Gender } & Male & 37 & 18 & 19 & \multirow{2}{*}{0.202} \\
\hline & Female & 12 & 7 & 5 & \\
\hline \multirow{2}{*}{ Age } & $<55$ & 20 & 10 & 10 & \multirow{2}{*}{0.777} \\
\hline & $\geq 55$ & 29 & 15 & 14 & \\
\hline \multirow{2}{*}{ AFP } & $<400 \mu \mathrm{g} / \mathrm{L}$ & 23 & 10 & 13 & \multirow{2}{*}{0.157} \\
\hline & $\geq 400 \mu \mathrm{g} / \mathrm{L}$ & 26 & 15 & 11 & \\
\hline \multirow{2}{*}{ TNM stage } & I & 16 & 6 & 10 & \multirow{2}{*}{0.004} \\
\hline & II + III & 33 & 19 & 14 & \\
\hline \multirow{2}{*}{ Tumor size $(\mathrm{cm})$} & $<5$ & 18 & 7 & 11 & \multirow{2}{*}{0.048} \\
\hline & $\geq 5$ & 31 & 18 & 13 & \\
\hline
\end{tabular}

Note. The median expression level was used as the cut-off. Low expression of miR-1290 in 24 patients was classified as values below the 50th percentile. High miR-1290 expression in 25 patients was classified as values at or above the 50th percentile. For analysis of correlation between miR-1290 expressions and clinical features, chi-square tests were used. Results were considered statistically significant at $p<0.05$

overexpression of SMEK1 in HUVECs could restore the mRNA and protein levels of SMEK1 in the presence of miR-1290 mimic. Moreover, rescue of SMEK1 expression significantly attenuated the phosphorylation of VEGFR2 upon treatment with miR-1290 mimic (Figure 6(b)). Importantly, tube formation, wound-healing, and migration assays further demonstrated that restoration of SMEK1 abolished miR-1290 mimic-induced endothelial functions in HUVECs (Figures 6(c)-6(e)). These results collectively implied that SMEK1 is a bona fide target of miR-1290 to mediate its role in promoting tumor angiogenesis and progression.

\section{Discussion}

Tumor angiogenesis is responsible for growth, invasion, and metastasis of HCC [2]. A growing body of evidence has suggested that the dysfunction of miRNAs in HCC affects tumor angiogenesis [22-25]. In the present study, we demonstrate that miR-1290 exerts a proangiogenic function through SMEK1. We show that miR-1290 downregulates SMEK1 expression at both the mRNA and protein levels. Moreover, miR-1290 inhibits the luciferase activity of wildtype but not the mutant $3^{\prime}$ UTR of SMEK1. By gain- and lossof-function analyses, we further show that SMEK1 can reduce the angiogenic ability of endothelial cells. Moreover, restoration of SMEK1 expression significantly abolishes miR-1290-induced VEGFR2 phosphorylation and the angiogenic phenotypes of endothelial cells in HCC. According to our in vivo xenograft experiments, administration of the miR-1290 antagomir effectively reduced the tumor angiogenesis and progression, providing evidence that targeting miR-1290 might be a potential strategy for angiogenesisbased cancer therapy.

MiR-1290 has been reported to be a maker of several human cancers, including pancreatic cancer, esophageal squamous cell carcinoma, breast cancer, colorectal cancer, and non-small-cell lung cancer [26-30]. These studies have shown that miR-1290 functions as an oncogene to promote tumor progression. For example, a miRNA array analysis indicates that the detection of elevated serum miR-1290 has the potential to improve the early detection of pancreatic cancer [31]. Another study reveals that miR-1290 acts as a crucial driver for tumor initiation and progression in human non-small-cell lung cancer [32]. However, the function of miR-1290 in HCC remains largely obscure. In the present study, we extend the study that miR-1290 was highly expressed in HCC patient serum-derived exosomes, tumor tissues, and HCC cell lines. Moreover, the ectopic miR-1290 was secreted from tumor cells into its surrounding microenvironment and delivered into endothelial cells via exosomes. In turn, miR-1290 downregulated SMEK1 expression and enhanced the phosphorylation of VEGFR2 in endothelial cells, eventually exacerbating HCC development by promoting tumor angiogenesis. Our results are consistent with the previous reports that SMEK1 controls endothelial cell function and subsequent angiogenesis by suppressing VEGFR2-mediated PI3 K/Akt/eNOS signaling pathway. [21].

Given that VEGF functions as a critical factor to promote vascular abnormalities in HCC, numerous researchers have focused on the dysregulated miRNAs that can target VEGF or other genes that alter the VEGF expression. For example, miR-195 is reported to suppress angiogenesis of HCC by directly inhibiting the expression of VEGF [33]. Moreover, miR-29c targets VEGFA to inhibit tumor angiogenesis of lung adenocarcinoma [34]. In this study, we described a novel pathway that tumor cells facilitated angiogenesis via secretion of exosomes containing miR-1290 into the surrounding microenvironment. Mechanistically, miR-1290 packed in exosomes were uptaken by recipient endothelial cells and subsequently downregulated SMEK1, thus resulting in enhanced tumor angiogenesis through a VEGFR2-mediated action. In line with this, tumor-derived exosomes have been shown to remodel the tumor and its metastatic environment [35-38]. Tumor cells can use exosomes as a cargo to transfer angiogenic factors, including proteins and miRNAs [39]. Recently, it has been demonstrated that exosomes released from human renal cancer stem cells trigger angiogenesis and form a prometastatic niche in lungs [40]. In line with these, our present study uncovered the function of secreted miR1290 as a critical mediator in the crosstalk between HCCs and endothelial cells. 


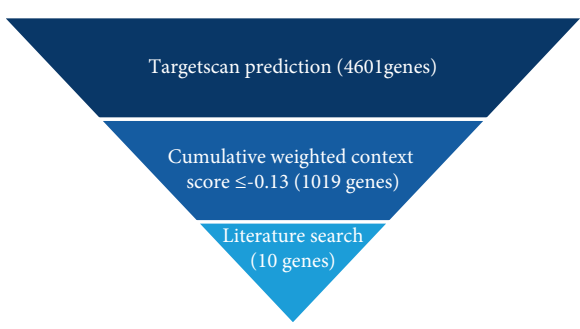

(a)

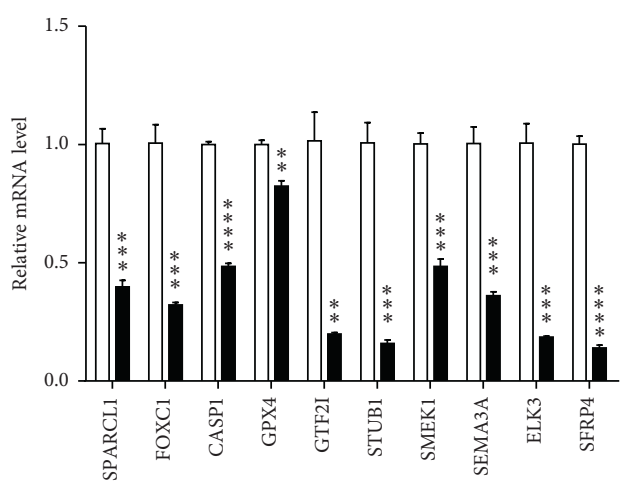

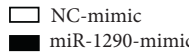

(b)
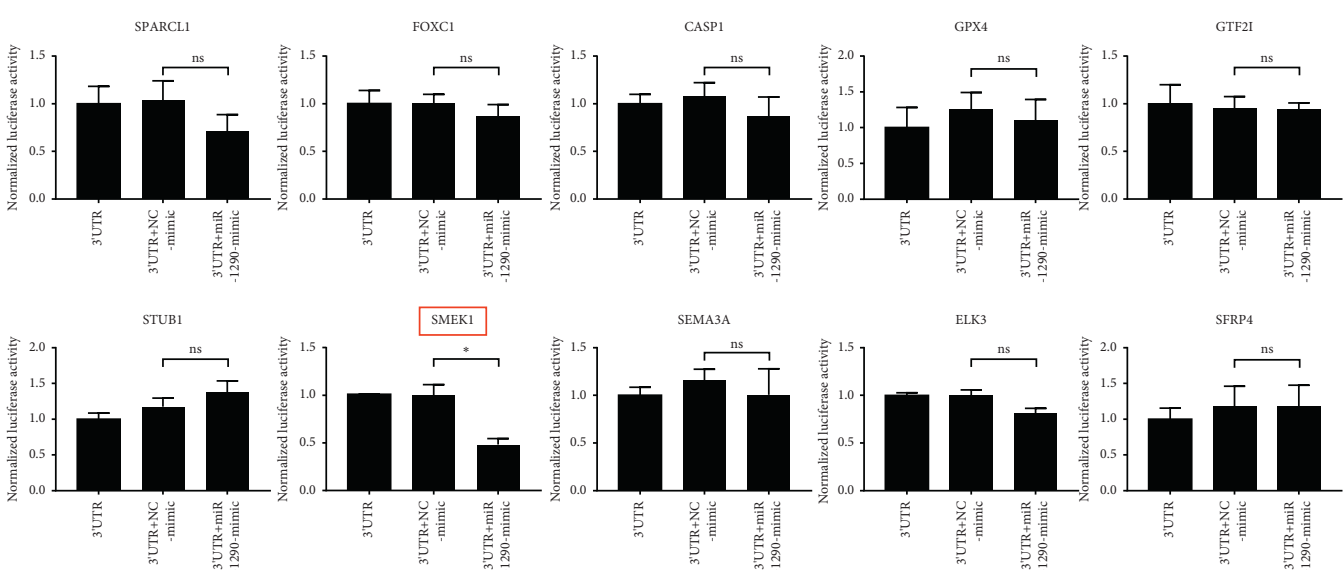

(c)
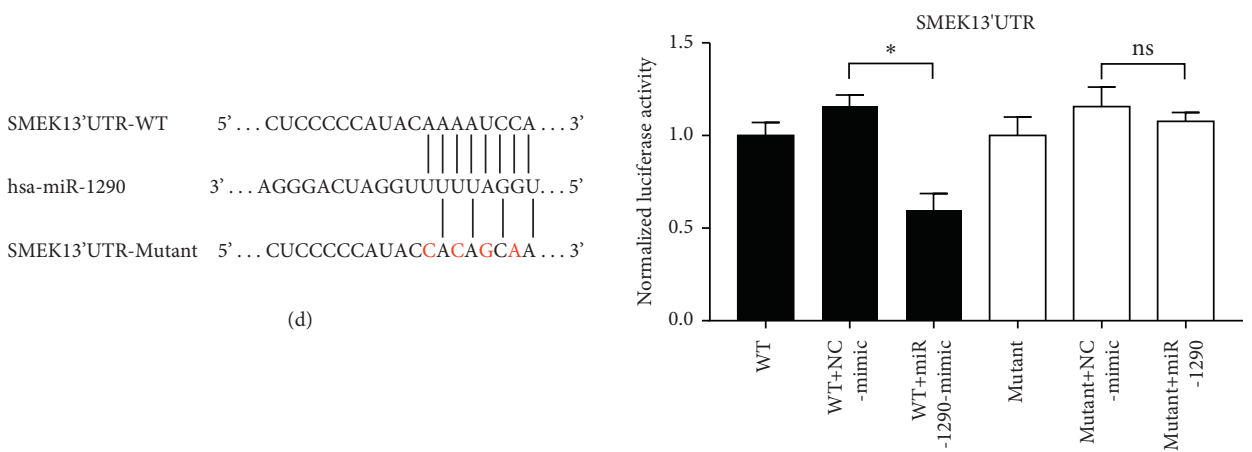

(e)
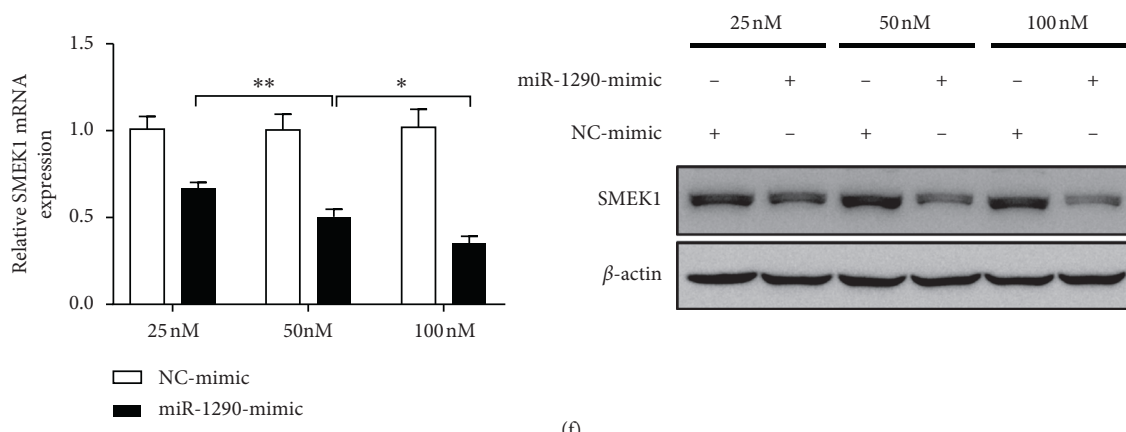

SMEKI

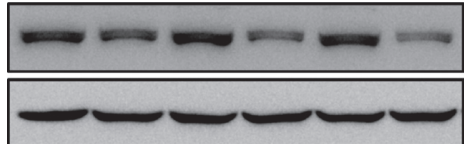

(f)

Figure 4: MiR-1290 targets SMEK1 directly in HUVECs. (a) Flow chart of screening miR-1290 target genes that were associated with angiogenesis. (b) The mRNA levels of 10 candidate genes in HUVECs that were transfected with miR-1290 mimic or NC mimic were detected by qRT-PCR. (c) HUVECs were cotransfected with miR-1290 mimic and the wild-type (WT) 3'UTR constructs of different candidate genes. Luciferase activities were determined $36 \mathrm{~h}$ after transfection. Luciferase values were normalized to Renilla activities. (d) The wild-type and mutant miR-1290 binding sites in the predicted target sequences of SMEK1 $3^{\prime} \mathrm{UTR}$ are indicated. (e) HUVECs were cotransfected with miR-1290 mimic and WT or mutant SMEK1 $3^{\prime}$ UTR constructs. Luciferase activities were determined $36 \mathrm{~h}$ after transfection. Luciferase values were normalized to Renilla activities. (f) HUVECs were transfected with different concentrations of miR1290 mimic for $48 \mathrm{~h}$. The expression of SMEK1 was detected by qRT-PCR and Western blotting. ${ }^{*} p<0.05 ;{ }^{* *} p<0.01 ;{ }^{* * *} p<0.001$; **** $p<0.0001$. Ns $=$ not significant. 


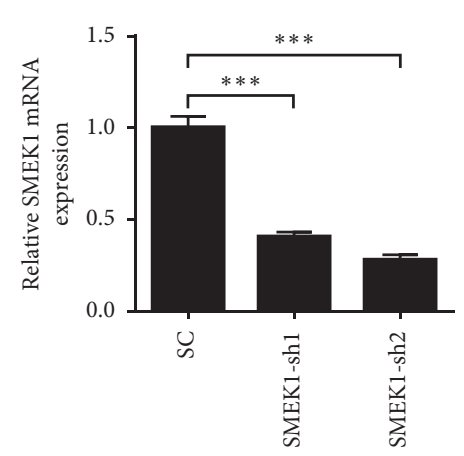

(a)

SC
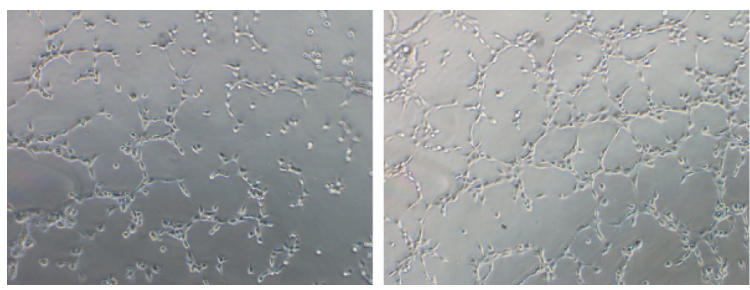

(c)
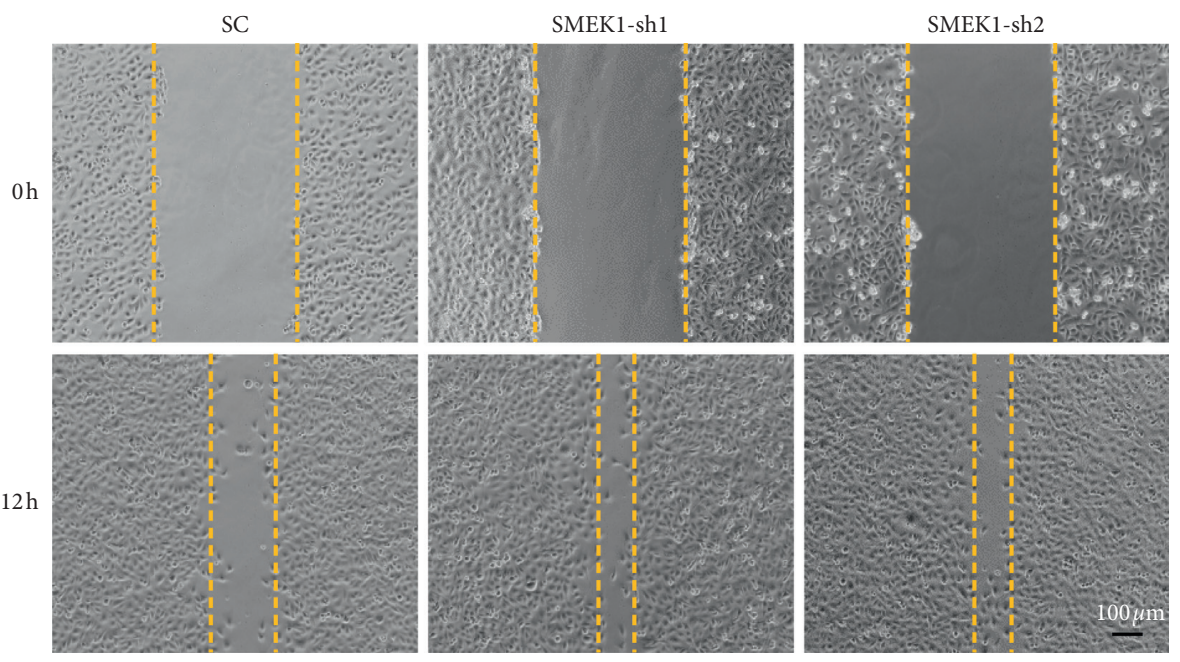

(d)
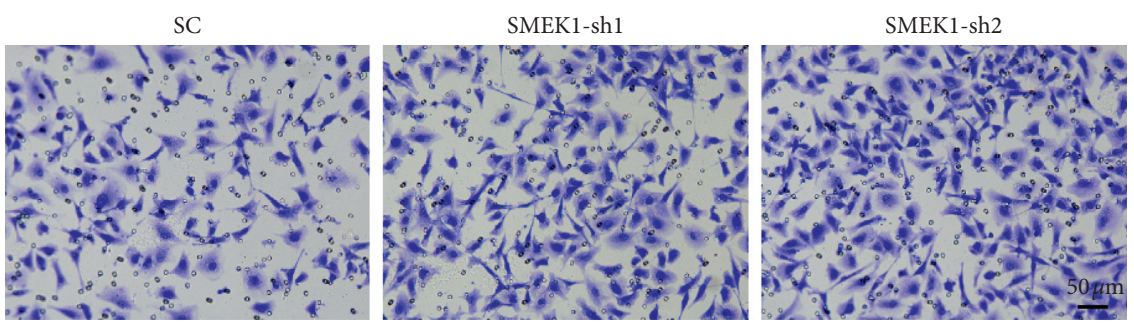

(e)
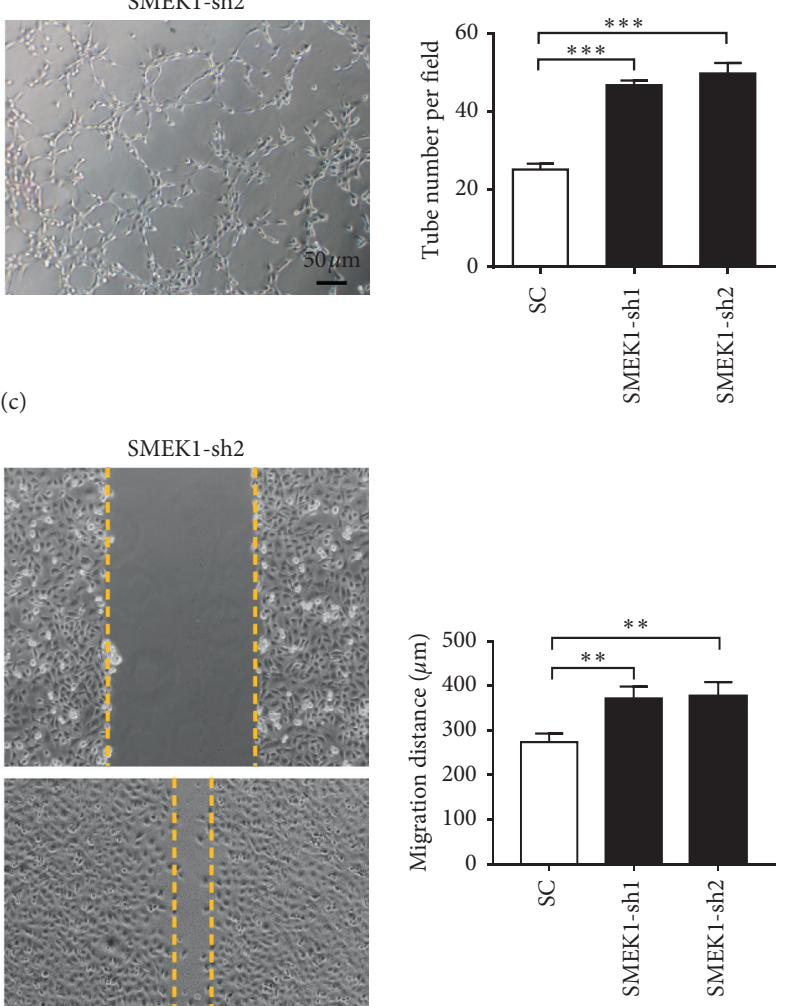

FIGURE 5: SMEK1 inhibits the angiogenic ability of endothelial cells. (a, b) Two independent shRNAs for SMEK1 were stably transfected into HUVECs via infection of lentivirus. The mRNA (a) and protein (b) level of SMEK1 were detected by qRT-PCR and Western blotting, respectively. (c, d, e) The tube formation assay (c), wound-healing assay (d), and Transwell assay (e) were performed in SMEK1-interfered HUVECs. ${ }^{* *} p<0.01 ;{ }^{* * *} p<0.001$. 


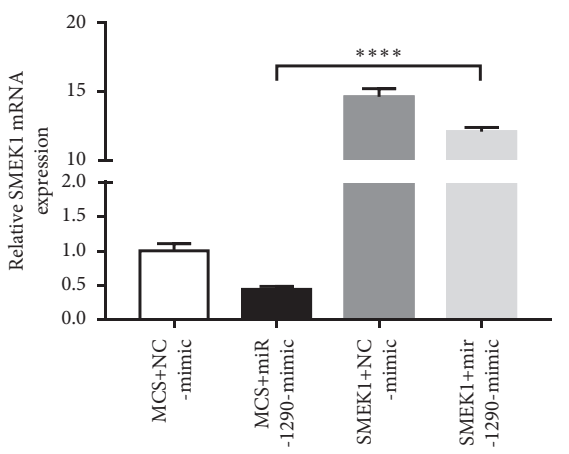

(a)

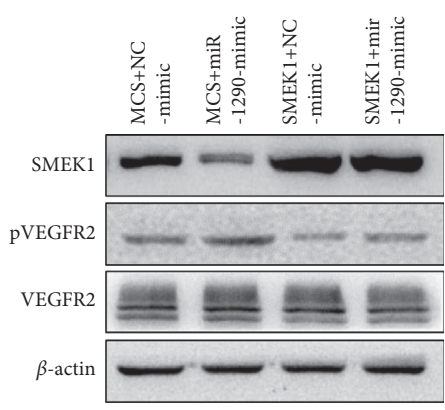

(b)

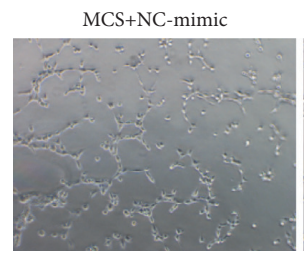

MCS+miR-1290-mimic

SMEK1+NC-mimic

SMEK1+miR-1290-mimic
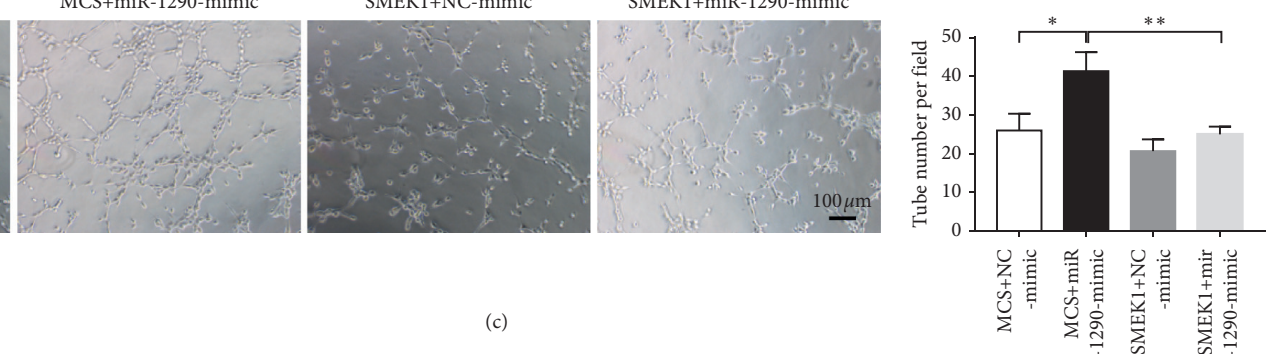

(c)
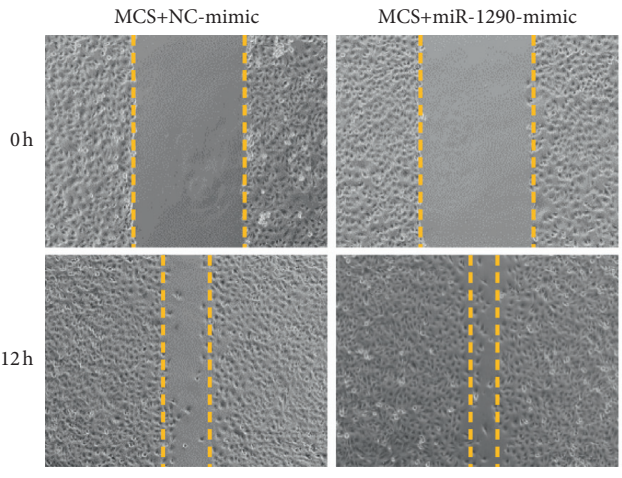

SMEK1+NC-mimic

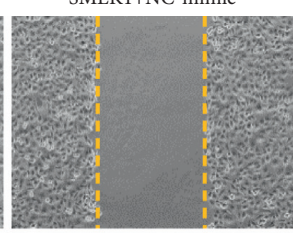

SMEK1+miR-1290-mimic
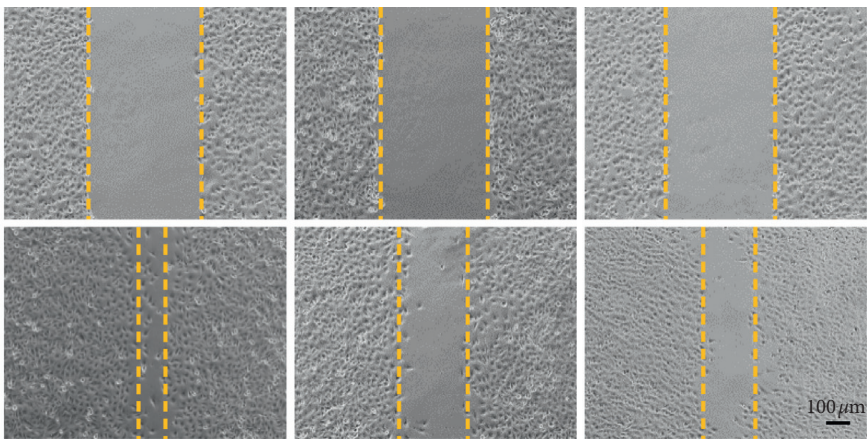

(d)
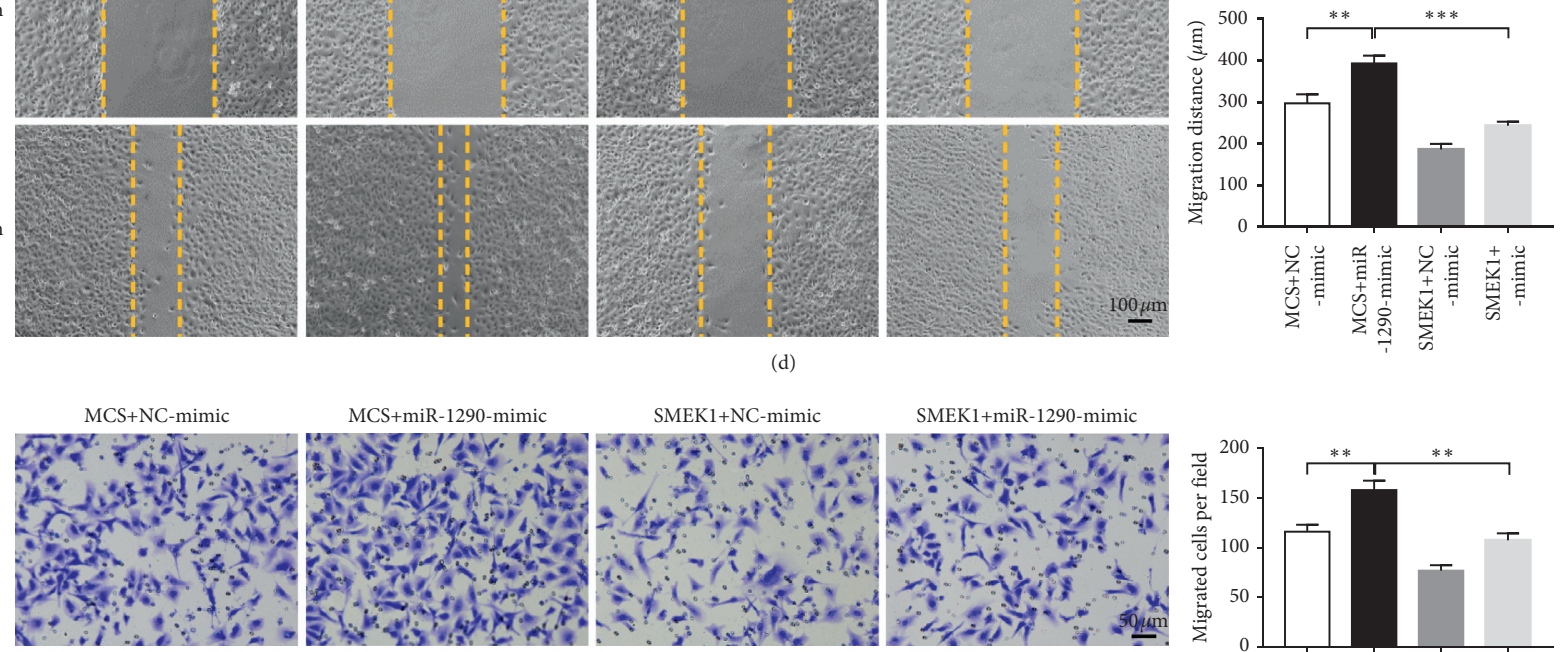

(e)

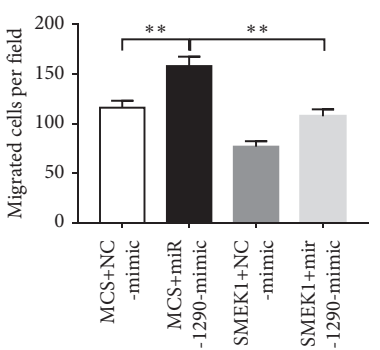

FIGURE 6: Rescue of SMEK1 expression attenuates the proangiogenic function of miR-1290. (a) SMEK1 were stably overexpressed in HUVECs in the presence or absence of miR-1290 mimic. The mRNA level of SMEK1 was detected by qRT-PCR. (b) The protein levels of SMEK1and pVEGFR2 were assessed by Western blotting. ( $c, d, e)$ The tube formation (c), wound-healing (d), and Transwell (e) assays were performed in SMEK1-expressing or control HUVECs in the presence or absence of miR-1290. ${ }^{*} p<0.05 ;{ }^{* *} p<0.01 ;{ }^{* * *} p<0.001$; ${ }^{* * * *} p<0.0001$. 


\section{Conclusions}

According to our data, mir-1290 is overexpressed in HCC patient serum-derived exosomes, and the delivery of miR1290 into human endothelial cells enhanced their angiogenic ability by alleviating the inhibition of VEGFR2 phosphorylation done by SMEK1. Our findings highlight the importance of exosomal miRNA-1290 in HCC angiogenesis, implicating miR-1290 as a potential therapeutic target for HCC.

\section{Data Availability}

The data used to support the findings of this study are available from the corresponding author upon request.

\section{Disclosure}

The manuscript has been presented as a preprint on https:// www.researchsquare.com/article/rs-3896/v2.

\section{Conflicts of Interest}

The authors declare that they have no conflicts of interest.

\section{Acknowledgments}

This work was supported by grants from the National Natural Science Foundation of China (nos. 81972454 and 81670600).

\section{Supplementary Materials}

Figure S1. miR-1290 targets SMEK1 inSMMC-7721 xenografts Table S1. A list of primers used in the reactions for qRT-PCR. Table S2. A list of primers used in the reactions for clone PCR. Table S3. MiRNA sequencing results. (Supplementary Materials)

\section{References}

[1] J. M. Llovet and J. Bruix, "Molecular targeted therapies in hepatocellular carcinoma," Hepatology, vol. 48, no. 4, pp. 1312-1327, 2008.

[2] M. Fernández, D. Semela, J. Bruix, I. Colle, M. Pinzani, and J. Bosch, "Angiogenesis in liver disease," Journal of Hepatology, vol. 50, no. 3, pp. 604-620, 2009.

[3] D. P. Bartel, "MicroRNAs: target recognition and regulatory functions," Cell, vol. 136, no. 2, pp. 215-233, 2009.

[4] A. Lujambio and S. W. Lowe, "The microcosmos of cancer," Nature, vol. 482, no. 7385, pp. 347-355, 2012.

[5] S.-T. Cha, P.-S. Chen, G. Johansson et al., "MicroRNA-519c suppresses hypoxia-inducible factor- $1 \alpha$ expression and tumor angiogenesis," Cancer Research, vol. 70, no. 7, pp. 2675-2685, 2010.

[6] L. Ma, J. Young, H. Prabhala et al., "miR-9, a MYC/MYCNactivated microRNA, regulates E-cadherin and cancer metastasis," Nature Cell Biology, vol. 12, no. 3, pp. 247-256, 2010.

[7] J.-H. Fang, H.-C. Zhou, C. Zeng et al., "MicroRNA-29b suppresses tumor angiogenesis, invasion, and metastasis by regulating matrix metalloproteinase 2 expression," Hepatology, vol. 54, no. 5, pp. 1729-1740, 2011.
[8] X. Yang, X.-F. Zhang, X. Lu et al., "MicroRNA-26a suppresses angiogenesis in human hepatocellular carcinoma by targeting hepatocyte growth factor-cMet pathway," Hepatology, vol. 59, no. 5, pp. 1874-1885, 2014.

[9] R. Bhome, F. Del Vecchio, G.-H. Lee et al., "Exosomal microRNAs (exomiRs): small molecules with a big role in cancer," Cancer Letters, vol. 420, pp. 228-235, 2018.

[10] L. Zhang, S. Zhang, J. Yao et al., "Microenvironment-induced PTEN loss by exosomal microRNA primes brain metastasis outgrowth," Nature, vol. 527, no. 7576, pp. 100-104, 2015.

[11] K. B. Challagundla, P. M. Wise, P. Neviani et al., "Exosome-mediated transfer of microRNAs within the tumor microenvironment and neuroblastoma resistance to chemotherapy," Journal of the National Cancer Institute, vol. 107, 2015.

[12] M. Y. Fong, W. Zhou, L. Liu et al., "Breast-cancer-secreted miR-122 reprograms glucose metabolism in premetastatic niche to promote metastasis," Nature Cell Biology, vol. 17, no. 2, pp. 183-194, 2015.

[13] R. M. Johnstone, M. Adam, J. R. Hammond, L. Orr, and C. Turbide, "Vesicle formation during reticulocyte maturation. Association of plasma membrane activities with released vesicles (exosomes)," Journal of Biological Chemistry, vol. 262, no. 19, pp. 9412-9420, 1987.

[14] J. Guduric-Fuchs, A. O’Connor, B. Camp, C. L. O’Neill, R. J. Medina, and D. A. Simpson, "Selective extracellular vesicle-mediated export of an overlapping set of microRNAs from multiple cell types," BMC Genomics, vol. 13, no. 1, p. 357, 2012.

[15] S. Manier, C.-J. Liu, H. Avet-Loiseau et al., "Prognostic role of circulating exosomal miRNAs in multiple myeloma," Blood, vol. 129, no. 17, pp. 2429-2436, 2017.

[16] R.-U. Takahashi, H. Miyazaki, and T. Ochiya, "The roles of MicroRNAs in breast cancer," Cancers, vol. 7, no. 2, pp. 598-616, 2015.

[17] T. Matsumura, K. Sugimachi, H. Iinuma et al., "Exosomal microRNA in serum is a novel biomarker of recurrence in human colorectal cancer," British Journal of Cancer, vol. 113, no. 2, pp. 275-281, 2015.

[18] X. Huang, T. Yuan, M. Liang et al., "Exosomal miR-1290 and miR-375 as prognostic markers in castration-resistant prostate cancer," European Urology, vol. 67, no. 1, pp. 33-41, 2015.

[19] X.-J. Lin, J.-H. Fang, X.-J. Yang et al., "Hepatocellular carcinoma cell-secreted exosomal MicroRNA-210 promotes angiogenesis in vitro and in vivo," Molecular Therapy-Nucleic Acids, vol. 11, pp. 243-252, 2018.

[20] J. Zhang, C. Zhou, H. Jiang et al., “ZEB1 induces ER-alpha promoter hypermethylation and confers antiestrogen resistance in breast cancer," Cell Death \& Disease, vol. 8, Article ID e2732, 2017.

[21] B.-R. Kim, S. H. Seo, M. S. Park, S.-H. Lee, Y. Kwon, and S. B. Rho, "sMEK1 inhibits endothelial cell proliferation by attenuating VEGFR-2-dependent-Akt/eNOS/HIF-1 $\alpha$ signaling pathways," Oncotarget, vol. 6, no. 31, pp. 31830-31843, 2015.

[22] T.-C. Shih, Y.-J. Tien, C.-J. Wen et al., "MicroRNA-214 downregulation contributes to tumor angiogenesis by inducing secretion of the hepatoma-derived growth factor in human hepatoma," Journal of Hepatology, vol. 57, no. 3, pp. 584-591, 2012.

[23] C. Du, X. Weng, W. Hu et al., "Hypoxia-inducible MiR-182 promotes angiogenesis by targeting RASA1 in hepatocellular carcinoma," Journal of Experimental \& Clinical Cancer Research, vol. 34, p. 67, 2015. 
[24] B. Zhou, R. Ma, W. Si et al., "MicroRNA-503 targets FGF2 and VEGFA and inhibits tumor angiogenesis and growth," Cancer Letters, vol. 333, no. 2, pp. 159-169, 2013.

[25] A. Ghosh, D. Dasgupta, A. Ghosh et al., "MiRNA199a-3p suppresses tumor growth, migration, invasion and angiogenesis in hepatocellular carcinoma by targeting VEGFA, VEGFR1, VEGFR2, HGF and MMP2," Cell Death \& Disease, vol. 8, Article ID e2706, 2017.

[26] A. E. Frampton, J. Krell, G. Kazemier, and E. Giovannetti, "Serum miR-1290 as a marker of pancreatic cancer-letter," Clinical Cancer Research, vol. 19, no. 18, pp. 5250-5251, 2013.

[27] Y. Mao, J. Liu, D. Zhang, and B. Li, "MiR-1290 promotes cancer progression by targeting nuclear factor I/X (NFIX) in esophageal squamous cell carcinoma (ESCC)," Biomedicine \& Pharmacotherapy, vol. 76, pp. 82-93, 2015.

[28] Y. Endo, T. Toyama, S. Takahashi et al., "miR-1290 and its potential targets are associated with characteristics of estrogen receptor $\alpha$-positive breast cancer," Endocrine-Related Cancer, vol. 20, no. 1, pp. 91-102, 2013.

[29] Q. Ma, Y. Wang, H. Zhang, and F. Wang, "miR-1290 contributes to colorectal cancer cell proliferation by targeting INPP4B," Oncology Research Featuring Preclinical and Clinical Cancer Therapeutics, vol. 26, no. 8, pp. 1167-1174, 2018.

[30] G. Kim, H.-J. An, M.-J. Lee et al., "Hsa-miR-1246 and hsamiR-1290 are associated with stemness and invasiveness of non-small cell lung cancer," Lung Cancer, vol. 91, pp. 15-22, 2016.

[31] A. Li, J. Yu, H. Kim et al., "MicroRNA array analysis finds elevated serum miR-1290 accurately distinguishes patients with low-stage pancreatic cancer from healthy and disease controls," Clinical Cancer Research, vol. 19, no. 13, pp. 3600-3610, 2013.

[32] W. C. Zhang, T. M. Chin, H. Yang et al., "Tumour-initiating cell-specific miR-1246 and miR-1290 expression converge to promote non-small cell lung cancer progression," Nature Communications, vol. 7, Article ID 11702, 2016.

[33] R. Wang, N. Zhao, S. Li et al., "MicroRNA-195 suppresses angiogenesis and metastasis of hepatocellular carcinoma by inhibiting the expression of VEGF, VAV2, and CDC42," Hepatology, vol. 58, no. 2, pp. 642-653, 2013.

[34] L. Liu, N. Bi, L. Wu et al., "MicroRNA-29c functions as a tumor suppressor by targeting VEGFA in lung adenocarcinoma," Molecular Cancer, vol. 16, p. 50, 2017.

[35] L. Milane, A. Singh, G. Mattheolabakis, M. Suresh, and M. M. Amiji, "Exosome mediated communication within the tumor microenvironment," Journal of Controlled Release, vol. 219, pp. 278-294, 2015.

[36] A. Hoshino, B. Costa-Silva, T.-L. Shen et al., "Tumour exosome integrins determine organotropic metastasis," Nature, vol. 527, no. 7578, pp. 329-335, 2015.

[37] H. Peinado, M. Alečković, S. Lavotshkin et al., "Melanoma exosomes educate bone marrow progenitor cells toward a pro-metastatic phenotype through MET," Nature Medicine, vol. 18, no. 6, pp. 883-891, 2012.

[38] A. Suetsugu, K. Honma, S. Saji, H. Moriwaki, T. Ochiya, and R. M. Hoffman, "Imaging exosome transfer from breast cancer cells to stroma at metastatic sites in orthotopic nudemouse models," Advanced Drug Delivery Reviews, vol. 65, no. 3, pp. 383-390, 2013.
[39] C. D'Souza-Schorey and J. W. Clancy, “Tumor-derived microvesicles: shedding light on novel microenvironment modulators and prospective cancer biomarkers," Genes \& Development, vol. 26, no. 12, pp. 1287-1299, 2012.

[40] C. Grange, M. Tapparo, F. Collino et al., "Microvesicles released from human renal cancer stem cells stimulate angiogenesis and formation of lung premetastatic niche," Cancer Research, vol. 71, no. 15, pp. 5346-5356, 2011. 